\title{
Genomic biomarkers for chronic kidney disease: the first step towards personalized medicine?
}

\author{
Jing-Yuan Cao', Le-Ting Zhou², Bi-Cheng Liu' ${ }^{1}$ \\ 'Institute of Nephrology, Southeast University School of Medicine, Nanjing 210009, China. \\ 2Department of Nephrology, Wuxi People's Hospital affiliated to Nanjing Medical University, Wuxi 214000, China.
}

Correspondence to: Dr. Bi-Cheng Liu, Institute of Nephrology, Southeast University School of Medicine, Nanjing 210009, China. E-mail: liubc64@163.com

How to cite this article: Cao JY, Zhou LT, Liu BC. Genomic biomarkers for chronic kidney disease: the first step towards personalized medicine? J Trans/ Genet Genom 2019;3:4. https://doi.org/10.20517/jtgg.2018.16

Received: 26 Jun 2018 First Decision: 20 Nov 2018 Revised: 9 Dec 2018 Accepted: 10 Dec 2018 Published: 20 Feb 2019

Science Editor: Sheng-Ying Qin Copy Editor: Cui Yu Production Editor: Huan-Liang Wu

\begin{abstract}
With the prevalence of end stage renal disease steadily increasing, chronic kidney disease (CKD) represents an impending public healthcare challenge. Classical diagnostic biomarkers of CKD, including creatinine, have low sensitivity and specificity. Thus, novel diagnostic and prognostic biomarkers for patients at high risk of early-stage progression are urgently needed. Personalized medicine approaches generally stratify patients according to their biological or genomic make-up. Targeted clinical trials require more precise identification of these subgroups. The use of new biomarkers obtained via high-throughput technologies is expected in future, accompanied by vast improvements in computational power applied in genomics, proteomics, and metabolomics studies using biological fluids and renal biopsy tissue. Genomic biomarkers may not only provide additional information regarding the etiology and mechanisms underlying CKD progression, but may also enable early diagnosis and the selection of appropriate drugs, thereby personalizing therapy. This review discusses commonly used research methods in genomic medicine and summarizes currently available genomic biomarkers in inherited and acquired CKD.
\end{abstract}

Keywords: Genomics, biomarkers, chronic kidney disease, personalized medicine, end-stage renal disease, high-throughput technology

\section{INTRODUCTION}

Chronic kidney disease (CKD), characterized by kidney damage and/or a decreased estimated glomerular filtration rate (eGFR) over a period of at least 3 months, imposes a drastic public health burden worldwide ${ }^{[1]}$. $\mathrm{CKD}$ of various origins commonly proceeds through the renal fibrosis pathway, resulting in end-stage renal 
disease (ESRD $)^{[1]}$. In 2015, 124,411 new patients were diagnosed with ESRD according to the United States Renal Data System, reflecting the increasing burden of kidney failure ${ }^{[2]}$. A possible explanation for increased renal failure is that the symptoms of most patients are non-specific or even missing in early-stage CKDs (stages 1 to 3). Therefore, timely diagnosis and treatment of patients at a high risk of progression in the early stage is a major clinical concern.

A biomarker is a quantifiable and analyzable characteristic that serves as an indicator of disease status, prognosis or response to therapeutic interventions ${ }^{[3]}$. Proteinuria and kidney function parameters are reliable and relatively non-invasive classical biomarkers; however, their sensitivity and specificity in detecting early renal injury is not optimal ${ }^{[4]}$. Moreover, they cannot provide pathological information at the molecular level. Therefore, there is an urgent need to uncover novel biomarkers with high sensitivity and specificity.

Personalized medical approaches generally stratify patients based on their biological or genomic makeup and thus optimize personalize management regimes. The genetic background has a strong influence on a wide spectrum of CKDs. Therefore, genomic biomarkers are expected to provide additional information regarding the etiology and mechanisms underlying CKD progression, as well as help identify more homogeneous patient subgroups and increasingly targeted therapies ${ }^{[5]}$. The use of new biomarkers obtained via high-throughput technologies is expected in the future, together with vast improvements in computational power applied to genomics, proteomics, and metabolomics studies using biological fluids and renal biopsy tissue ${ }^{[6]}$. So far, such studies have focused on optimzing development of new therapies, drug selection and drug dosage ${ }^{[7]}$.

The aim of personalized medicine is to enable clinicians to rapidly, efficiently and accurately determine the most appropriate diagnosis and therapy for a patient ${ }^{[8]}$. Genomic biomarkers may not only provide information regarding the etiology and mechanisms underlying CKD progression but may also be used for early diagnosis and for the selection of appropriate drugs, thereby personalizing therapy.

In this review, we first introduce commonly used genomic medicine methods. Then, we summarize the potential genomic biomarker candidates of inherited and acquired CKD. Finally, we discuss the opportunities and challenges offered by personalized genomic medicine.

\section{OVERVIEW OF GENE VARIATIONS AND GENETIC TESTING}

According to databases including Gencode, Ensembl and RefSeq, there are approximately 19,000 to 21,000 protein-coding genes in the human genome ${ }^{[9]}$. Moreover, all humans share approximately $99.9 \%$ similarity in their DNA ${ }^{[10]}$.

Nucleotide-level variations occur frequently in the human genome, the most common being a single-nucleotide polymorphism (SNP), which is a variation in a single nucleotide between genome sequences. Laboratories use high-throughput whole genome sequencing for genotyping of all SNPs in an individual. SNPs are present at $>1 \%$ within a given population and account for a majority of normal human genetic variations ${ }^{[11]}$. Most SNPs have no obvious phenotypic effects, although some can affect gene expression and susceptibility to certain diseases $^{[12]}$. Earlier methods for genome screening for genetic etiologies of disease employed candidate gene studies and linkage analysis. In 2005, the first genome-wide association study (GWAS) was performed ${ }^{[13]}$. Since then, GWASs have emerged as the most widely used tools to map risk-associated loci for complex pathologies via analysis of the association between genome-wide markers and the disease.

Even though genetic predisposition of an individual is a critical factor in determining renal disease manifestation, robust interactions between genetic predisposition and environmental factors strongly influence the course of CKD. Only a few renal disorders, such as Alport syndrome (AS) and autosomal dominant polycystic kidney disease (ADPKD), are caused by single-gene variations. Such disorders can 
be diagnosed via mutation analysis, and their clinical manifestations are similar among patients, although variants in other genes may influence or modify disease course and severity ${ }^{[14]}$.

For multifactorial kidney diseases with complex causal relationships and poorly understood mechanisms of action, such as diabetic nephropathy (DN) and hypertensive nephrosclerosis, disease course and the rate of progression to ESRD vary substantially among patients. Over the past decade, rapid advances in genetics have raised expectations for a switch from traditional medical diagnosis and treatment towards personalized medicine. Numerous genetic variants have been implicated in studies of CKD. Herein, we review the current genomic biomarkers in inherited and acquired CKD.

\section{EMERGING GENOMIC BIOMARKERS FOR INHERITED CKD}

\section{AS}

AS is an inherited disease caused by mutations affecting collagen type IV (specifically, COL4A3 and COL4A4 on chromosome 2 and COL4A5 on chromosome $\mathrm{X}$ ) in the glomerular basement membrane (GBM). It is characterized by kidney damage, hearing loss, and eye abnormalities ${ }^{[15,16]}$. Almost all patients experience hematuria and proteinuria at symptom onset. AS occurs in approximately 1 in 50,000 neonates and men are more likely to be symptomatic than women ${ }^{[17]}$. Moreover, it accounts for $>1 \%$ of patients receiving renal replacement therapy ${ }^{[15]}$. Genetic testing has generally replaced rather invasive procedures such as kidney or skin biopsy, and can yield an accurate diagnosis in approximately $95 \%$ of AS patients ${ }^{[18]}$.

In $80 \%-85 \%$ of cases, AS is inherited in an X-linked manner (XLAS) and is caused by mutations in COL $4 A 5$. Additional, inheritance patterns include autosomal recessive AS (ARAS) manner, or, less commonly, autosomal dominant AS (ADAS) owing to mutations in COL4A3 or COL4A4 ${ }^{[19]}$.

Cervera-Acedo et al. ${ }^{[19]}$ assessed a Spanish family with variable phenotypes of ADAS via clinical, histological, and genetic analyses. They reported that carriers of p.G333E and p.P1461L or p.S1492C mutations in COL4A3 presented an earlier onset of disease than individuals, who carried only the p.G333E mutation. Fu et al. ${ }^{[20]}$ reported that a synonymous p.Gly292Gly mutation in XLAS could alter the splicing donor site of COL4A5.

Targeted capture and next-generation sequencing allowed Liu et al. ${ }^{[21]}$ to detect 15 novel mutations, 6 known mutations, as well as 2 novel fragment deletions in the genomes of 20 patients with AS from unrelated Chinese families. Weber et al. ${ }^{[22]}$ identified 47 novel mutations in AS and thin basement membrane nephropathy (TBMN) patients through an assessment of 216 individuals. Guo et al. ${ }^{[23]}$ reported that the intron mutation c.4127+11C $>\mathrm{T}$ and missense mutation c.4195A $>\mathrm{T}$ in COL4A4 were possible causes of ADAS [Table 1].

These results broadened our understanding of mutations in 3 different collagen type IV genes, which have important implications as genomic biomarkers in diagnosis, prognosis, and genetic counseling. Further studies are expected to elucidate the correlations between these novel identified genetic mutations and the resulting phenotypes.

\section{TBMN}

Collagen type IV-related nephropathies include another disorder closely resembling AS, referred to as TBMN. Also called benign familial hematuria, TBMN is characterized by persistent hematuria, minimal proteinuria, normal renal function, and a uniformly thinner $\mathrm{GBM}^{[24,25]}$. Despite the fact that TBMN does not require treatment, its exact diagnosis is still important because its clinical findings overlap with those of early AS.

In contrast to AS, TBMN is usually caused by heterozygous mutations of COL4A3 or COL4A4 with the carrier having ARAS. Several novel mutations of these two genes have been reported as pathogenic in recent years. Hou et al. ${ }^{[26]}$ reported that a novel mutation $(3725 \mathrm{G}>\mathrm{A}, \mathrm{G} 1242 \mathrm{D})$ in COL4A3 resulted in TBMN 
Table 1. Genome-wide association studies of inherited chronic kidney disease

\begin{tabular}{|c|c|c|c|c|}
\hline Diseases & Sample size & Study population & Main findings & References \\
\hline AS & A family & Spanish & $\begin{array}{l}\text { In ADAS, carriers of p.G333E and p.P1461L or p.S1492C mutations in COL4A3 } \\
\text { have an earlier onset of disease than those of only p.G333E mutation }\end{array}$ & [19] \\
\hline AS & A family & Japanese & $\begin{array}{l}\text { A synonymous p.Gly292Gly mutation in XLAS can alter the splicing donor site of } \\
\text { the COL4A5 gene }\end{array}$ & {$[20]$} \\
\hline AS & 20 patients & Chinese & Detected 15 novel mutations and 6 known mutations & [21] \\
\hline AS & 216 patients & European & Identified 47 novel mutations in AS and TBMN & [22] \\
\hline AS & A family & Chinese & $\begin{array}{l}\text { The intron mutation c. } 4127+11 \mathrm{C}>\mathrm{T} \text { and missense mutation c. } 4195 \mathrm{~A}>\mathrm{T} \text { in } \mathrm{CO} \angle 4 A 4 \\
\text { were possible causes of ADAS }\end{array}$ & [23] \\
\hline TBMN & A family & Chinese & $\begin{array}{l}\text { A novel mutation ( } 3725 \mathrm{G}>\mathrm{A}, \mathrm{G} 1242 \mathrm{D} \text { ) in COL4A3 resulted in TBMN } \\
\text { pathogenesis }\end{array}$ & {$[26]$} \\
\hline TBMN & A family & Chinese & Identified a novel heterozygous splicing mutation in $\operatorname{COL4A4}(\mathrm{c} .1459+1 \mathrm{G}>\mathrm{A})$ & [27] \\
\hline TBMN & 45 patients & Korean & $\begin{array}{l}\text { Identified } 2 \text { potential pathogenic variants, G199R and G1606E, along with another } \\
6 \text { novel variants }\end{array}$ & [28] \\
\hline TBMN & A family & Cypriot & Mutations in COLAA1 collagen type IV $\alpha 1$ chains could also cause TBMN & [29] \\
\hline ADPKD & A family & Chinese & $\begin{array}{l}\text { Identify a novel heterozygous frameshift mutation c.3976_3977insCT } \\
\text { (p.F1326Sfs`21) in PKD1 }\end{array}$ & [30] \\
\hline ADPKD & A family & Chinese & Reported a novel frameshift mutation, c. 12605_12632del28, in PKD1 & [35] \\
\hline
\end{tabular}

AS:Alport syndrome; ADAS: autosomal dominant AS; XLAS: X-linked AS; TBMN: thin basement membrane nephropathy; ADPKD: autosomal dominant polycystic kidney disease

pathogenesis within an identical family. Xu et al. ${ }^{[27]}$ identified a novel heterozygous splicing mutation in COL4A4 (c.1459+1G>A), which might be responsible for TBMN. Baek et al. ${ }^{[2]}$ investigated the sequence of full-length COL4A4 in 45 Korean TBMN patients and identified 2 potential pathogenic variants, G199R and G1606E, along with another 6 novel variants. Interestingly, mutations in other genes may still contribute to TBMN. Gale et al. ${ }^{[29]}$ performed genome-wide linkage analysis, whole-exome sequencing, and co-segregation analyses on 20 family members and reported that mutations in COL4A1 collagen type IV $\alpha 1$ chains could also cause TBMN [Table 1]. Given these findings, a combination of genetic testing and immunofluorescence analysis appears most suitable to guarantee an exact diagnosis.

\section{ADPKD}

$\mathrm{ADPKD}$ is the most prevalent monogenic renal disease. It is characterized by the development of renal cysts, which chronically impair kidney structure and function. It is estimated that approximately $50 \%$ of patients with ADPKD eventually develop ESRD ${ }^{[30]}$. ADPKD is reportedly associated with mutations in polycystinencoding genes $P K D 1$ and $P K D 2$, with $P K D 1$ mutations having been reported in up to $85 \%$ of patients ${ }^{[31]}$. In 2016, the rare third gene encoding glucosidase II alpha subunit (GANAB) at position 11q12.3, was reported and estimated to account for $0.3 \%$ of cases $^{[32,33]}$.

To date, more than 1,200 and 200 pathogenic germline mutations in $P K D 1$ and $P K D 2$, have been archived in the Mayo PKD database ${ }^{[34]}$, and the numbers are still increasing. For instance, targeted exome sequencing of $P K D 1$ and $P K D 2$, allowed Sha et al. ${ }^{[30]}$ to identify a novel heterozygous frameshift mutation c.3976_3977insCT (p.F1326Sfs ${ }^{\star} 21$ ) in PKD1. Wang et al ${ }^{[35]}$ reported a novel frameshift mutation, c.12605_12632del28, in PKD1 in a Chinese family with ADPKD [Table 1].

Genetic testing is currently applied to assess patients with atypical radiologic presentations or a negative family history. With decreasing costs and faster sequencing speed, its clinical use may be expanded to indicate prognosis and guide patient management in the near future.

\section{EMERGING GENOMIC BIOMARKERS FOR ACQUIRED CKD}

\section{Idiopathic membranous nephropathy}

Idiopathic membranous nephropathy (IMN) is an immune complex-mediated disease and represents a common cause of glomerulonephritis. Gene sequencing and GWASs have yielded major breakthroughs regarding IMN biomarkers in recent years. 
Stanescu et al ${ }^{[36]}$ reported two loci, phospholipase A2 receptor 1 (PLA2R1) and human leukocyte antigen (HLA)-DQA1, strongly associated with IMN risk among 556 European patients. The associations were further strengthened when the two loci were evaluated simultaneously. These findings are consistent with the fact that the PLA2R1-encoded protein, M-type PLA2R1, is the major autoantigen in humans with IMN. Moreover, a large cross-sectional study involving over 2000 Chinese individuals detected anti-PLA2R antibodies in $73 \%$ of subjects, who carried both risk alleles but none in those with no mutation in these two genes, suggesting that the interaction between PLA2R1 and HLA-DQA1 variants contributes to the presence of anti-PLA2R antibodies ${ }^{[37]}$. Interestingly, Sekula et al ${ }^{[38]}$ reported that mutations in PLA2R1 were specific to IMN, whereas $H L A-D Q A 1$ variants (encoding major histocompatibility complex, class II, DQ alpha 1) were also associated with other kidney diseases including type $1 \mathrm{DN}$, lupus nephritis and focal segmental glomerular sclerosis (FSGS) in adults. By sequencing the entire major histocompatibility complex region in over 200 DNA samples, Le et al. ${ }^{[39]}$ reported a strong association between PLA2R-related IMN and HLA$\mathrm{DRB}^{*}{ }^{\star}$ 5:01 and HLA-DRB3 ${ }^{*} 02: 02$ alleles in the Chinese population. How exactly mutations in PLA2R finally result in the generation of anti-PLA2R antibodies remains to be elucidated [Table 2].

Anti-PLA2R1 antibodies are detected in nearly $70 \%$ of patients with IMN. Measurement of anti-PLA2R antibodies has been translated into clinical practice for diagnosing IMN, and emerging studies indicate it can also be used to predict the response to immunosuppressive therapy and long-term outcomes ${ }^{[40]}$. Considering that genetic mutation is an upstream event, detection of risk variants might provide early diagnostic and prognostic information on $\mathrm{IMN}^{[41,42]}$. However, long-term prospective studies are still required to establish the statistical likelihood of individuals with high-risk genotypes developing IMN over their lifetime.

\section{IgA nephropathy}

IgA nephropathy (IgAN) is a major cause of renal failure worldwide. IgAN perfectly exemplifies how multiple gene interactions can affect kidney injury because the combined distribution of risk-associated alleles strongly correlates with its geographic prevalence gradient; it is highest in Asian, intermediate in European, and lowest in African countries ${ }^{[43]}$. In the past 5 years, several reviews and meta-analyses have discussed GWAS findings for $\operatorname{IgAN}^{[44-48]}$.

The increasing number of larger GWASs in different populations has unveiled numerous IgAN-associated loci, including complement factor H-related $(C F H R)$ genes ${ }^{[49-51]}$, or those encoding defensing $(D E F A)^{[47,52]}$, $H L A^{[53]}$, sprouty RTK signaling antagonist $2(S P R Y 2)^{[54]}$, vav guanine nucleotide exchange factor $3(V A V 3)^{[46]}$, core 1 synthase, glycoprotein- $N$-acetylgalactosamine 3-beta-galactosyltransferase $1(C 1 G A L T 1)^{[55]}$, and tumor

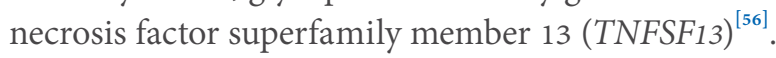

In 2010, Feehally et al. ${ }^{[53]}$ reported a genome-wide analysis in an IgAN cohort selected from the UK Glomerulonephritis DNA Bank. Their results suggested that the HLA locus contained the strongest common susceptibility alleles responsible for genetic predisposition to IgAN in the European population. Factor $\mathrm{H}$, a component of the alternative pathway, is present in the mesangial immune deposits, which can be activated by IgA1, and contributes to IgAN progression ${ }^{[57]}$. Gharavi et al.$^{[49]}$ performed targeted follow-up evaluations in Chinese and European cohorts including 1,950 patients and 1,920 controls, and detected a common deletion in CFHR1 and CFHR3 at position 1q32 and a locus at position 22q12 in IgAN individuals. Deletions in CFHR1 and CFHR3 were related to a reduced risk of IgAN. In contrast, Jullien et al. ${ }^{[50]}$ reported deletion variants of CFHR1 and CFHR3, which were associated with mesangial immune deposits but not with IgAN progression. Zhai et al. ${ }^{[51]}$ recruited 500 IgAN patients and 576 healthy controls and sequenced all exons, intronic flanking regions, and the untranslated regions of CFHR5. They reported 32 variants in CFHR5 (including 28 rare and 4 common variants). Rare variants in CFHR5 may further increase the genetic predisposition to IgAN, which indicates that CFHR5 is a susceptibility gene for IgAN. 
Table 2. Genome-wide association studies of acquired chronic kidney disease

\begin{tabular}{|c|c|c|c|c|}
\hline Diseases & Sample size & Study population & Main findings & References \\
\hline IMN & 556 patients and 2388 controls & European & $\begin{array}{l}\text { Two loci, PLA2R1 and HLA-DQA1, strongly associated } \\
\text { with IMN risk }\end{array}$ & {$[36]$} \\
\hline IMN & 1112 patients and 1020 controls & Chinese & $\begin{array}{l}\text { The interaction between } P L A 2 R 1 \text { and } H L A-D Q A 1 \text { variants } \\
\text { contribute to the presence of anti-PLA2R antibodies }\end{array}$ & [37] \\
\hline IMN & 149 patients and 100 controls & Chinese & $\begin{array}{l}\text { HLA-DRB } 1^{\star} 15: 01 \text { and } \mathrm{HLA}-\mathrm{DRB} 3^{\star} 02: 02 \text { alleles } \\
\text { independently and strongly associate with PLA2R-related } \\
\text { IMN }\end{array}$ & [39] \\
\hline $\lg A N$ & $\begin{array}{l}244 \text { patients, } 4,980 \text { healthy } \\
\text { individuals, } 186 \text { families }\end{array}$ & European & $\begin{array}{l}\text { HLA locus contained the strongest common susceptibility } \\
\text { alleles responsible for genetic predisposition to IgAN in } \\
\text { the European population }\end{array}$ & [53] \\
\hline $\operatorname{IgAN}$ & $\begin{array}{l}1,950 \text { patients and } 1,920 \\
\text { controls }\end{array}$ & $\begin{array}{l}\text { Chinese and } \\
\text { European }\end{array}$ & $\begin{array}{l}\text { Deletions in CFHR1 and CFHR3 were related to a reduced } \\
\text { risk of IgAN }\end{array}$ & [49] \\
\hline $\lg A N$ & 500 patients and 576 controls & Chinese & CFHR5 is a susceptibility gene for IgAN & [51] \\
\hline $\lg A N$ & 20612 participants & $\begin{array}{l}\text { European and East } \\
\text { Asian }\end{array}$ & $\begin{array}{l}\text { Identified } 6 \text { new risk loci: } 2 \text { new independent signals at } \\
H L A-D Q B 1 \text { and DEFA; plus } 4 \text { in /TGAM-ITGAX, VAV3, } \\
\text { and CARD9 }\end{array}$ & [46] \\
\hline $\lg A N$ & $\begin{array}{l}8,313 \text { patients and } 19,680 \\
\text { controls }\end{array}$ & Han Chinese & $\begin{array}{l}\text { The allelic frequencies of the variants within } S T 6 G A L 1 \text {, } \\
A C C S \text {, and } D E F A \text { correlated with geographical variations } \\
\text { in IgAN prevalence }\end{array}$ & [47] \\
\hline $\lg A N$ & $\begin{array}{l}1000 \text { IgAN cases and } 1000 \\
\text { controls }\end{array}$ & Chinese & $\begin{array}{l}\text { Genetic variations and gene expression levels of TNFSF13 } \\
\text { were related to the susceptibility and severity of IgAN } \\
\text { among the Han population }\end{array}$ & [56] \\
\hline DN & $\begin{array}{l}5,825 \text { with diabetes and } 46,061 \\
\text { without diabetes }\end{array}$ & European & $\begin{array}{l}\text { SNPs in HS6ST1 and RAB38/CTSC exerted a genetic } \\
\text { effect on albuminuria only in individuals with diabetes }\end{array}$ & {$[75]$} \\
\hline DN & 743 patients and 646 controls & European & $\begin{array}{l}\text { Loci } 11 p 15.4 \text {, near the CARS gene, and } 13 \mathrm{q} 33.3 \\
\text { encompassing an intergenic region between MYO16 and } \\
\text { IRS2 genes, were susceptible to kidney disease in both } \\
\text { type } 1 \text { and } 2 \text { diabetes }\end{array}$ & {$[76]$} \\
\hline DN & 3,652 patients & Finnish & $\begin{array}{l}\text { rs } 4972593 \text { on chromosome 2q31.1 was a sex-specific } \\
\text { genetic variant related to ESRD in patients with type } 1 \\
\text { diabetes }\end{array}$ & {$[77]$} \\
\hline DN & 406 patients and 214 controls & Chinese & rs2796498 might be associated with DN & [79] \\
\hline
\end{tabular}

IMN: idiopathic membranous nephropathy; IgAN: IgA nephropathy; DN: diabetic nephropathy; SNP: single-nucleotide polymorphism; ESRD: end-stage renal disease

Kiryluk et al ${ }^{[46]}$ performed a follow-up evaluation in 20,612 European and East Asian individuals. They identified 6 new risk loci: 2 new independent signals at HLA-DQB1 and DEFA; plus 4 in ITGAM-ITGAX (encoding integrin subunits $\mathrm{M}$ and $\mathrm{X}$ ), VAV3, and CARD9 (encoding caspase recruitment domain family member 9). Li et al. ${ }^{[4]}$ conducted a GWAS comprising 8,313 patients and 19,680 controls. They reported that the allelic frequencies of the variants within ST6GAL1 (encoding ST6 beta-galactoside alpha-2,6sialyltransferase 1), ACCS (encoding 1-aminocyclopropane-1-carboxylate synthase homolog), and DEFA correlated with geographical variations in IgAN prevalence. Milillo et al. ${ }^{[54]}$ reported that a SPRY2 mutation inhibited the mitogen-associated protein kinase/extracellular signal-related kinase pathway, which was associated with an autosomal dominant form of IgAN. Gale et al ${ }^{[55]}$ reported that a common variation in C1GALT1 influenced galactose-deficient IgA1 levels in the population, which was independently associated with the risk of progressive IgAN. Finally, Zhong et al. ${ }^{[56]}$ reported that genetic variations and gene expression levels of TNFSF 13 were related to the susceptibility and severity of IgAN among the Han population [Table 2].

Overall, these studies emphasize complex multilocus model for IgAN. Multiple rare variants participating in a common network can influence disease susceptibility. Future investigations might explore the development of combined biomarkers to generate a prognostic model which can usher into potential genomic biomarkers and drug targets for personalized therapy ${ }^{[58]}$.

\section{Apolipoprotein L1-related kidney disease}

The incidence of ESRD varies substantially between African and European individuals ${ }^{[59]}$. 
Rare mutations in $\mathrm{MYH} 9$ (encoding myosin heavy chain 9) can cause monogenic diseases with kidney involvement, referred to collectively as Epstein-Fechtner syndrome, and thus represent candidates for ESRD diagnosis. The locus associated with ESRD was narrowed down subsequently filtered to three functional sequence variants in the nearby apolipoprotein L1 (APOL1) gene ${ }^{[60,61]}$. Risk-associated variants of APOL1 (G1 and G2) are strongly linked to human immunodeficiency virus (HIV) associated nephropathy, FSGS, and CKD progression among African individuals ${ }^{[62]}$. They are considered to display such high frequencies because of a selection event primarily attributed to providing protection from Trypanosoma brucei rhodesiense $e^{[63,64]}$.

A large number of follow-up studies have been carried out following identification of APOL1 variants, which represent a milestone in kidney disease genetics. In the African American Study of Kidney Disease and Hypertension, $58.1 \%$ of patients in the APOL1 high-risk group were diagnosed with ESRD or had double the serum creatinine level. In contrast, only $36.6 \%$ of those in the APOL1 low-risk group reached the primary outcome $e^{[65]}$.

In the Chronic Renal Insufficiency Cohort study, patients from the APOL1 high-risk group experienced a more rapid decline in eGFR and an increased risk of the composite renal outcome than Caucasians ${ }^{[65]}$. In recent years, the APOL1-related disease spectrum was extended to systemic lupus erythematosus-associated glomerulopathy ${ }^{[66]}$, proteinuria ${ }^{[67]}$, and HIV-associated nephropathy ${ }^{[68]}$; it was not, however, investigated among African individuals with $\operatorname{IgAN}^{[69]}$. Taken together, these data suggest that detection of APOL1 variants may serve as a prognostic marker for CKD progression.

As an additional benefit, APOL1 genotyping could improve safety and success of kidney transplantation and facilitate the match of kidney donors with receivers. Freedman et al ${ }^{[70]}$ reported a strong association between the risk-associated APOL1 genotype of the kidney donor and renal allograft failure. However, the genotype of the allograft recipient does not seem to affect allograft survival ${ }^{[71]}$. These findings raise a critical issue: should kidneys from donors with an APOL1 risk-associated genotype be used for transplantation ${ }^{[72]}$ ? To address this question, the new APOL1 Long-term Kidney Transplantation Outcomes Network is set to perform a large multicenter cohort study and evaluate the risk exposed by APOL1 genotyping to both donors and recipients.

\section{DN}

Diabetes-related complications represent one of the most severe public healthcare challenges worldwide, with great social and economic burden ${ }^{[73]}$. DN is a prominent complication of diabetes and DN, the primary cause of ESRD. Moreover, it is highly heritable, with an incidence of approximately $35 \%$ in type 1 diabetes ${ }^{[74]}$. Nevertheless, the identification of gene variants strongly associated with DN has been limited.

The onset of albuminuria is regarded as an early landmark of DN progression. By performing meta-analyses of GAWSs and subsequent validation in diabetic and nondiabetic populations, Teumer et al ${ }^{[7]}$ identified associations between variants of cubilin-encoding CUBN and albuminuria in the overall population, whereas SNPs in HS6ST1 and RAB38/CTSC exerted a genetic effect on albuminuria only in individuals with diabetes. Pezzolesi et al. ${ }^{[76]}$ performed a trans-ethnic meta-analysis of data from the Japanese and Genetics of Kidneys in Diabetes collections. They reported that loci 11p15.4, near the cysteinyl-tRNA synthetase (CARS) gene, and 13q33.3 encompassing an intergenic region between the myosin XVI (MYO16) and insulin receptor substrate 2 (IRS2) genes, were susceptible to kidney disease in both type 1 and 2 diabetes. Sandholm et al. ${ }^{[77]}$ performed a GWAS in 3,652 patients from the Finnish Diabetic Nephropathy (FinnDiane) study. They suggested that rs4972593 on chromosome 2 q31.1 was a sex-specific genetic variant related to ESRD in patients with type 1 diabetes and may provide sex-specific protection against ESRD. A variant of SCAF8, encoding SR-related CTD associated factor 8, was consistently associated with type $2 \mathrm{DN}$ in 
different populations ${ }^{[78]}$. Genotyping of 406 type 2 diabetes patients and 214 controls from the Chinese Han population by Li et al. ${ }^{[79]}$ revealed rs10789038 and rs2796498 polymorphisms in adenosine monophosphateactivated protein kinase subunit alpha 2 (PRKAA2), which were related to susceptibility to type 2 diabetes, whereas rs2796498 might be associated with DN [Table 2].

However, a large, comprehensive meta-GWAS effort was unable to identify clear loci associated with $\mathrm{DN}$ and many of the previously identified candidate signals were not validated ${ }^{[74]}$. Such apparent lack of reproducibility may be explained by differences in study design, populations, outcome ascertainment, and/or false-positive results between different studies ${ }^{[80]}$. Overall, these results indicate that the genetic landscape of $\mathrm{DN}$ is more complex than expected. An increasing number of large, diverse population-based studies on DN are required to provide conclusive genomic evidence.

\section{TOWARDS PERSONALIZED MEDICINE: FUTURE PROSPECTS AND CHALLENGES}

Personalized genomic medicine promises to combine genomic data with clinical phenotypes to develop novel clinical biomarkers for predicting CKD risk, drug selection, and for accurate monitoring of patient prognosis. To achieve this goal, numerous obstacles must be overcome, including determination of the most significant genetic markers, limiting the off-target effects of gene-based therapies, and conducting clinical studies to confirm genetic variants associated with drug response ${ }^{[7]}$.

Questions regarding personalized medicine have been summarized by Joyner and Paneth $^{[81]}$. One of the most important issues regarding what types of studies should be performed for personalized medicine because convenient samples have often been used without considering how selection bias and other factors could influence the outcome ${ }^{[81]}$. The Secretary's Advisory Committee on Genetic Testing had proposed four criteria to guide the assessment of benefits and risks with a genetic test: (1) analytical validity; (2) clinical validity; (3) clinical utility; and (4) social consequences. Strategies complying with these recommendations are required to obtain a panel of genomic biomarkers for diagnosis, prognostic evaluation, and genotypeguided counseling $^{[82]}$.

Even though mRNA expression levels are not necessarily a functional read-out, we previously reported that urinary podocalyxin, $\mathrm{CD} 2$-associated protein, $\alpha$-actin4, and podocin mRNAs correlated with serum creatinine in DN patients ${ }^{[83]}$. Using targeted microarrays, we identified urinary vimentin mRNA as a biomarker to predict renal fibrosis and verified its predictive ability in CKD patients ${ }^{[84]}$. Upon iterative random forest analysis of a targeted microarray, four fibrosis-associated mRNAs (tumor growth factor $\beta 1$, matrix metallopeptidase 9 , tissue inhibitor of metalloproteinases 2 , and vimentin) in urinary sediments were identified as sensitive predictors of tubulointerstitial fibrosis ${ }^{[85]}$.

Despite compelling examples of the use of genomics to support personalized medicine, genomics alone is unlikely to provide sufficient information regarding disease pathophysiology and prognosis. Indeed, in spite of other omics approaches, including transcriptomics and metabolomics, have emerged as powerful tools for developing novel biomarkers for CKD in recent years ${ }^{[86-88]}$, and proteomics remains the classic realm for biomarker discovery. In this respect, transcriptional, translational, and post-translational modifications, which cause functional changes to proteins and their function, represent another unexplored area. To maximize the information obtained by these various approaches, integrative personal omics profiling (iPOP) is increasingly regarded as a promising strategy that combines genomic, transcriptomic, proteomic (including autoantibodies), and metabolomic profiles from the same individual for long-term follow-up of their genomic/transcriptomic composition ${ }^{[89]}$. Longitudinal iPOP is extremely powerful in interpreting healthy and diseased states as it associates genomic information with other dynamic omics activity. 


\section{CONCLUSION}

To date, there exist numerous potential genomic biomarkers of inherited and acquired CKD. Some of them have been utilized in clinical practice to improve diagnostic efficiency and to predict the response to immunosuppressive therapy as well as long-term outcomes of CKD patients. However, personalized medicine does not immediately provide a permanent solution for patient management. Further refinements in the application of personalized medicine are required to focus on genomics and other omics. Meticulous, large, multicenter, and cost-effective genomic studies are required to validate the potential candidates indicated herein, and novel genomic biomarkers for CKD are awaiting identification.

\section{DECLARATIONS}

\section{Authors' contributions}

Conceived the review: Cao JY, Liu BC

Wrote the paper: Cao JY, Zhou LT

Edited and revised manuscript: Cao JY, Zhou LT, Liu BC

Approved final version of manuscript: Liu BC

\section{Availability of data and materials}

Not applicable.

\section{Financial support and sponsorship}

This work was supported by the National Key R\&D Program of China (2018YFC1314000), the National Natural Science Foundations of China $(81470997,81670696,81720108007)$ and the Medical Science and Technology Support Project of Jiangsu Province (BL2014080).

\section{Conflicts of interest}

All authors declared that there are no conflicts of interest.

\section{Ethical approval and consent to participate}

Not applicable.

\section{Consent for publication}

Not applicable.

\section{Copyright}

(C) The Author(s) 2019.

\section{REFERENCES}

1. Webster AC, Nagler EV, Morton RL, Masson P. Chronic kidney disease. Lancet 2017;389:1238-52.

2. Benjamin O, Lappin SL. End-stage renal disease. Treasure Island (FL): StatPearls Publishing; 2018.

3. Biomarkers Definitions Working Group. Biomarkers and surrogate endpoints: preferred definitions and conceptual framework. Clin Pharmacol Ther 2001;69:89-95.

4. Urbschat A, Obermuller N, Haferkamp A. Biomarkers of kidney injury. Biomarkers 2011;16 Suppl 1:S22-30.

5. Wuttke M, Köttgen A. Insights into kidney diseases from genome-wide association studies. Nat Rev Nephrol 2016;12:549-62.

6. Schena FP. Biomarkers and personalized therapy in chronic kidney diseases. Expert Opin Investig Drugs 2014;23:1051-4.

7. Hamburg MA, Collins FS. The path to personalized medicine. N Engl J Med 2010;363:301-4.

8. Aronson SJ, Rehm HL. Building the foundation for genomics in precision medicine. Nature 2015;526:336-42.

9. Salzberg SL. Open questions: how many genes do we have? BMC Biol 2018;16:94.

10. Venter JC, Smith HO, Adams MD. The sequence of the human genome. Clin Chem 2015;61:1207-8.

11. Hansen TF, Jakobsen A. Clinical implications of genetic variations in the VEGF system in relation to colorectal cancer. Pharmacogenomics 2011;12:1681-93.

12. Chakravarti A. To a future of genetic medicine. Nature 2001;409:822-3. 
13. Klein RJ, Zeiss C, Chew EY, Tsai JY, Sackler RS, et al. Complement factor H polymorphism in age-related macular degeneration. Science 2005;308:385-9.

14. Nordfors L, Luttropp K, Carrero JJ, Witasp A, Stenvinkel P, et al. Genetic studies in chronic kidney disease: basic concepts. J Nephrol 2012;25:141-9.

15. Gross O, Kashtan CE, Rheault MN, Flinter F, Savige J, et al. Advances and unmet needs in genetic, basic and clinical science in Alport syndrome: report from the 2015 International Workshop on Alport Syndrome. Nephrol Dial Transplant 2017;32:916-24.

16. Kashtan CE, Ding J, Garosi G, Heidet L, Massella L, et al. Alport syndrome: a unified classification of genetic disorders of collagen IV

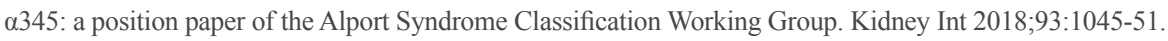

17. Watson S, Bush JS. Alport syndrome. Treasure Island (FL): StatPearls Publishing; 2018.

18. Savige J, Gregory M, Gross O, Kashtan C, Ding J, et al. Expert guidelines for the management of Alport syndrome and thin basement membrane nephropathy. J Am Soc Nephrol 2013;24:364-75.

19. Cervera-Acedo C, Coloma A, Huarte-Loza E, Sierra-Carpio M, Domínguez-Garrido E. Phenotype variability in a large Spanish family with Alport syndrome associated with novel mutations in COL4A3 gene. BMC Nephrol 2017;18:325.

20. Fu XJ, Nozu K, Eguchi A, Nozu Y, Morisada N, et al. X-linked Alport syndrome associated with a synonymous p.Gly292Gly mutation alters the splicing donor site of the type IV collagen alpha chain 5 gene. Clin Exp Nephrol 2016;20:699-702.

21. Liu JH, Wei XX, Li A, Cui YX, Xia XY, et al. Novel mutations in COL4A3, COL4A4, and COL4A5 in Chinese patients with Alport Syndrome. PLoS One 2017;12:e0177685.

22. Weber S, Strasser K, Rath S, Kittke A, Beicht S, et al. Identification of 47 novel mutations in patients with Alport syndrome and thin basement membrane nephropathy. Pediatr Nephrol 2016;31:941-55.

23. Guo L, Li D, Dong S, Wan D, Yang B, et al. Mutation analysis of COL4A3 and COL4A4 genes in a Chinese autosomal-dominant Alport syndrome family. J Genet 2017;96:389-92.

24. Savige J, Rana K, Tonna S, Buzza M, Dagher H, et al. Thin basement membrane nephropathy. Kidney Int 2003;64:1169-78.

25. Tryggvason K, Patrakka J. Thin basement membrane nephropathy. J Am Soc Nephrol 2006;17:813-22.

26. Hou P, Chen Y, Ding J, Li G, Zhang H. A novel mutation of COL4A3 presents a different contribution to Alport syndrome and thin basement membrane nephropathy. Am J Nephrol 2007;27:538-44.

27. Xu Y, Guo M, Dong H, Jiang W, Ma R, et al. A novel COL4A4 mutation identified in a Chinese family with thin basement membrane nephropathy. Sci Rep 2016;6:20244.

28. Baek JI, Choi SJ, Park SH, Choi JY, Kim CD, et al. Identification of novel variants in the COL4A4 gene in Korean patients with thin basement membrane nephropathy. Indian J Med Res 2009;129:525-33.

29. Gale DP, Oygar DD, Lin F, Oygar PD, Khan N, et al. A novel COL4A1 frameshift mutation in familial kidney disease: the importance of the C-terminal NC1 domain of type IV collagen. Nephrol Dial Transplant 2016;31:1908-14.

30. Sha YK, Sha YW, Mei LB, Huang XJ, Wang X, et al. Use of targeted sequence capture and high-throughput sequencing identifies a novel PKD1 mutation involved in adult polycystic kidney disease. Gene 2017;634:1-4.

31. Harris PC, Rossetti S. Molecular diagnostics for autosomal dominant polycystic kidney disease. Nat Rev Nephrol 2010;6:197-206.

32. Porath B, Gainullin VG, Cornec-Le Gall E, Dillinger EK, Heyer CM, et al. Mutations in GANAB, encoding the glucosidase II $\alpha$ subunit, cause autosomal-dominant polycystic kidney and liver disease. Am J Hum Genet 2016;98:1193-1207.

33. Cornec-Le Gall E, Torres VE, Harris PC. Genetic complexity of autosomal dominant polycystic kidney and liver diseases. J Am Soc Nephrol 2018;29:13-23.

34. Song X, Haghighi A, Iliuta IA, Pei Y. Molecular diagnosis of autosomal dominant polycystic kidney disease. Expert Rev Mol Diagn 2017; 17:885-95.

35. Wang Z, Wang Y, Xiong J. A new PKD1 mutation discovered in a Chinese family with autosomal polycystic kidney disease. Kidney Blood Press Res 2014;39:1-8.

36. Stanescu HC, Arcos-Burgos M, Medlar A, Bockenhauer D, Kottgen A, et al. Risk HLA-DQA1 and PLA(2)R1 alleles in idiopathic membranous nephropathy. N Engl J Med 2011;364:616-26.

37. Lv J, Hou W, Zhou X, Liu G, Zhou F, et al. Interaction between PLA2R1 and HLA-DQA1 variants associates with anti-PLA2R antibodies and membranous nephropathy. J Am Soc Nephrol 2013;24:1323-9.

38. Sekula P, Li Y, Stanescu HC, Wuttke M, Ekici AB, et al. Genetic risk variants for membranous nephropathy: extension of and association with other chronic kidney disease aetiologies. Nephrol Dial Transplant 2017;32:325-32.

39. Le WB, Shi JS, Zhang T, Liu L, Qin HZ, et al. HLA-DRB1*15:01 and HLA-DRB3*02:02 in PLA2R-related membranous nephropathy. J Am Soc Nephrol 2017;28:1642-50.

40. Bullich G, Ballarín J, Oliver A, Ayasreh N, Silva I, et al. HLA-DQA1 and PLA2R1 polymorphisms and risk of idiopathic membranous nephropathy. Clin J Am Soc Nephrol 2014;9:335-43.

41. Kanigicherla D, Gummadova J, McKenzie EA, Roberts SA, Harris S, et al. Anti-PLA2R antibodies measured by ELISA predict long-term outcome in a prevalent population of patients with idiopathic membranous nephropathy. Kidney Int 2013;83:940-8.

42. Ronco P, Debiec H. Membranous nephropathy: a fairy tale for immunopathologists, nephrologists and patients. Mol Immunol 2015;68:57-62.

43. Kiryluk K, Li Y, Sanna-Cherchi S, Rohanizadegan M, Suzuki H, et al. Geographic differences in genetic susceptibility to IgA nephropathy: GWAS replication study and geospatial risk analysis. PLoS Genet 2012;8:e1002765.

44. Kiryluk K, Novak J. The genetics and immunobiology of IgA nephropathy. J Clin Invest 2014;124:2325-32.

45. Kiryluk K, Novak J, Gharavi AG. Pathogenesis of immunoglobulin A nephropathy: recent insight from genetic studies. Annu Rev Med 2013;64:339-56.

46. Kiryluk K, Li Y, Scolari F, Sanna-Cherchi S, Choi M, et al. Discovery of new risk loci for IgA nephropathy implicates genes involved in 
immunity against intestinal pathogens. Nat Genet 2014;46:1187-96.

47. Li M, Foo JN, Wang JQ, Low HQ, Tang XQ, et al. Identification of new susceptibility loci for IgA nephropathy in Han Chinese. Nat Commun 2015;6:7270.

48. Nie R, Cheng G, Zhang J, Dong Y, Wang C, et al. The association of rs1047763 and rs1008898 of C1GALT1 with IgA nephropathy risk: a global meta-analysis. Monoclon Antib Immunodiagn Immunother 2017;36:95-103.

49. Gharavi AG, Kiryluk K, Choi M, Li Y, Hou P, et al. Genome-wide association study identifies susceptibility loci for IgA nephropathy. Nat Genet 2011;43:321-7.

50. Jullien P, Laurent B, Claisse G, Masson I, Dinic M, et al. Deletion variants of CFHR1 and CFHR3 associate with mesangial immune deposits but not with progression of IgA nephropathy. J Am Soc Nephrol 2018;29:661-9.

51. Zhai YL, Meng SJ, Zhu L, Shi SF, Wang SX, et al. Rare variants in the complement factor H-related protein 5 gene contribute to genetic susceptibility to IgA nephropathy. J Am Soc Nephrol 2016;27:2894-905.

52. Qi YY, Zhou XJ, Cheng FJ, Hou P, Zhu L, et al. DEFA gene variants associated with IgA nephropathy in a Chinese population. Genes Immun 2015;16:231-7.

53. Feehally J, Farrall M, Boland A, Gale DP, Gut I, et al. HLA has strongest association with IgA nephropathy in genome-wide analysis. J Am Soc Nephrol 2010;21:1791-7.

54. Milillo A, La Carpia F, Costanzi S, D’Urbano V, Martini M, et al. A SPRY2 mutation leading to MAPK/ERK pathway inhibition is associated with an autosomal dominant form of IgA nephropathy. Eur J Hum Genet 2015;23:1673-8.

55. Gale DP, Molyneux K, Wimbury D, Higgins P, Levine AP, et al. Galactosylation of IgA1 is associated with common variation in C1GALT1. J Am Soc Nephrol 2017;28:2158-66.

56. Zhong Z, Feng SZ, Xu RC, Li ZJ, Huang FX, et al. Association of TNFSF13 polymorphisms with IgA nephropathy in a Chinese Han population. J Gene Med 2017; doi: 10.1002/jgm.2966.

57. Maillard N, Wyatt RJ, Julian BA, Kiryluk K, Gharavi A, et al. Current understanding of the role of complement in IgA nephropathy. J Am Soc Nephrol 2015;26:1503-12.

58. Cox SN, Pesce F, El-Sayed Moustafa JS, Sallustio F, Serino G, et al. Multiple rare genetic variants co-segregating with familial IgA nephropathy all act within a single immune-related network. J Intern Med 2017;281:189-205.

59. Xue JL, Eggers PW, Agodoa LY, Foley RN, Collins AJ. Longitudinal study of racial and ethnic differences in developing end-stage renal disease among aged medicare beneficiaries. J Am Soc Nephrol 2007;18:1299-306.

60. Kopp JB, Smith MW, Nelson GW, Johnson RC, Freedman BI, et al. MYH9 is a major-effect risk gene for focal segmental glomerulosclerosis. Nat Genet 2008;40:1175-84.

61. Tzur S, Rosset S, Shemer R, Yudkovsky G, Selig S, et al. Missense mutations in the APOL1 gene are highly associated with end stage kidney disease risk previously attributed to the MYH9 gene. Hum Genet 2010;128:345-50.

62. Limou S, Nelson GW, Kopp JB, Winkler CA. APOL1 kidney risk alleles: population genetics and disease associations. Adv Chronic Kidney Dis 2014;21:426-33.

63. Genovese G, Friedman DJ, Ross MD, Lecordier L, Uzureau P, et al. Association of trypanolytic ApoL1 variants with kidney disease in African Americans. Science 2010;329:841-5.

64. Limou S, Dummer PD, Nelson GW, Kopp JB, Winkler CA. APOL1 toxin, innate immunity, and kidney injury. Kidney Int 2015;88:28-34.

65. Parsa A, Kao WH, Xie D, Astor BC, Li M, et al. APOL1 risk variants, race, and progression of chronic kidney disease. N Engl J Med 2013;369:2183-96.

66. Larsen CP, Beggs ML, Saeed M, Walker PD. Apolipoprotein L1 risk variants associate with systemic lupus erythematosus-associated collapsing glomerulopathy. J Am Soc Nephrol 2013;24:722-5.

67. Lipkowitz MS, Freedman BI, Langefeld CD, Comeau ME, Bowden DW, et al. Apolipoprotein L1 gene variants associate with hypertensionattributed nephropathy and the rate of kidney function decline in African Americans. Kidney Int 2013;83:114-20.

68. Kopp JB, Nelson GW, Sampath K, Johnson RC, Genovese G, et al. APOL1 genetic variants in focal segmental glomerulosclerosis and HIVassociated nephropathy. J Am Soc Nephrol 2011;22:2129-37.

69. Papeta N, Kiryluk K, Patel A, Sterken R, Kacak N, et al. APOL1 variants increase risk for FSGS and HIVAN but not IgA nephropathy. J Am Soc Nephrol 2011;22:1991-6.

70. Freedman BI, Pastan SO, Israni AK, Schladt D, Julian BA, et al. APOL1 genotype and kidney transplantation outcomes from deceased African American donors. Transplantation 2016;100:194-202.

71. Lee BT, Kumar V, Williams TA, Abdi R, Bernhardy A, et al. The APOL1 genotype of African American kidney transplant recipients does not impact 5-year allograft survival. Am J Transplant 2012;12:1924-8.

72. Chandraker A. The real world impact of APOL1 variants on kidney transplantation. Transplantation 2016;100:16-7.

73. Cooper ME. Diabetes: treating diabetic nephropathy-still an unresolved issue. Nat Rev Endocrinol 2012;8:515-6.

74. Sandholm N Van Zuydam N, Ahlqvist E, Juliusdottir T, Deshmukh HA, et al. The genetic landscape of renal complications in type 1 diabetes. J Am Soc Nephrol 2017;28:557-74.

75. Teumer A, Tin A, Sorice R, Gorski M, Yeo NC, et al. Genome-wide association studies identify genetic loci associated with albuminuria in diabetes. Diabetes 2016;65:803-17.

76. Pezzolesi MG, Poznik GD, Skupien J, Smiles AM, Mychaleckyj JC, et al. An intergenic region on chromosome 13q33.3 is associated with the susceptibility to kidney disease in type 1 and 2 diabetes. Kidney Int 2011;80:105-11.

77. Sandholm N, McKnight AJ, Salem RM, Brennan EP, Forsblom C, et al. Chromosome 2q31.1 associates with ESRD in women with type 1 diabetes. J Am Soc Nephrol2013;24:1537-43. 
78. Iyengar SK, Sedor JR, Freedman BI, Kao WH, Kretzler M, et al. Genome-wide association and trans-ethnic meta-analysis for advanced diabetic kidney disease: family investigation of nephropathy and diabetes (FIND). PLoS Genet 2015;11:e1005352.

79. Li Q, Li C, Li H, Zeng L, Kang Z, et al. Effect of AMP-activated protein kinase subunit alpha 2 (PRKAA2) genetic polymorphisms on susceptibility to type 2 diabetes mellitus and diabetic nephropathy in a Chinese population. J Diabetes 2018;10:43-9.

80. Limou S, Vince N, Parsa A. Lessons from CKD-related genetic association studies-moving forward. Clin J Am Soc Nephrol 2018;13:140-52.

81. Joyner MJ, Paneth N. Seven questions for personalized medicine. JAMA 2015;314:999-1000.

82. Witasp A, Nordfors L, Carrero JJ, Luttropp K, Lindholm B, et al. Genetic studies in chronic kidney disease: interpretation and clinical applicability. J Nephrol 2012;25:851-64.

83. Zheng M, Lv LL, Ni J, Ni HF, Li Q, et al. Urinary podocyte-associated mRNA profile in various stages of diabetic nephropathy. PLoS One 2011;6:e20431.

84. Cao YH, Lv LL, Zhang X, Hu H, Ding LH, et al. Urinary vimentin mRNA as a potential novel biomarker of renal fibrosis. Am J Physiol Renal Physiol 2015;309:F514-22.

85. Zhou LT, Cao YH, Lv LL, Ma KL, Chen PS, et al. Feature selection and classification of urinary mRNA microarray data by iterative random forest to diagnose renal fibrosis: a two-stage study. Sci Rep 2017;7:39832.

86. Breit M, Weinberger KM. Metabolic biomarkers for chronic kidney disease. Arch Biochem Biophys 2016;589:62-80.

87. Hocher B, Adamski J. Metabolomics for clinical use and research in chronic kidney disease. Nat Rev Nephrol 2017;13:269-84.

88. Lyu LL, Feng Y, Liu BC. Urinary biomarkers for chronic kidney disease with a focus on gene transcript. Chin Med J (Engl) 2017;130:2251-6.

89. Chen R, Mias GI, Li-Pook-Than J, Jiang L, Lam HY, et al. Personal omics profiling reveals dynamic molecular and medical phenotypes. Cell 2012;148:1293-307. 